\title{
Molecular Epigenetics and Genetics in Neuro-Oncology
}

\author{
Raman P. Nagarajan and Joseph F. Costello \\ Brain Tumor Research Center, Department of Neurosurgery, Helen Diller Family Comprehensive Cancer Center, \\ University of California San Francisco, San Francisco, \\ California 94143
}

\begin{abstract}
Summary: Gliomas arise through genetic and epigenetic alterations of normal brain cells, although the exact cell of origin for each glioma subtype is unknown. The alteration-induced changes in gene expression and protein function allow uncontrolled cell division, tumor expansion, and infiltration into surrounding normal brain parenchyma. The genetic and epigenetic alterations are tumor subtype and tumor-grade specific. Particular alterations predict tumor aggressiveness, tumor response to therapy, and patient survival. Genetic alterations include deletion, gain, amplification, mutation, and translocation, which result in oncogene activation and tumor suppressor gene inactivation, or in some instances the alterations may simply be a consequence of tumorigenesis. Epigenetic alterations in brain tumors include $\mathrm{CpG}$ island hypermethylation associated with tumor suppressor gene silencing, gene-specific hypomethylation associated with aberrant gene activation, and genome-wide
\end{abstract}

hypomethylation potentially leading to loss of imprinting, chromosomal instability, and cellular hyperproliferation. Other epigenetic alterations, such as changes in the position of histone variants and changes in histone modifications are also likely to be important in the molecular pathology of brain tumors. Given that histone deacetylases are targets for drugs that are already in clinical trial, surprisingly little is known about histone acetylation in primary brain tumors. Although a majority of epigenetic alterations are independent of genetic alterations, there is interaction on specific genes, signaling pathways and within chromosomal domains. Next-generation sequencing technology is now the method of choice for genomic and epigenome profiling, allowing more comprehensive understanding of genetic and epigenetic contributions to tumorigenesis in the brain. Key Words: Genomics, epigenomics, gliomas, methylation, acetylation.

\section{GENE REGULATION BY EPIGENETIC MECHANISMS}

Epigenetics is defined as mitotically heritable changes in gene expression that are not due to changes in the primary DNA sequence. Epigenetic mechanisms include enzymatic modification of DNA and associated histone proteins that regulate and maintain gene expression states, and have important roles in chromosome structure and stability. The discovery of altered epigenetic profiles in human neoplasia has led to a new paradigm in which both genetic and epigenetic mechanisms contribute significantly to cancer and perhaps many other common human diseases. Because of their reversible nature, epigenetic alterations are being targeted therapeutically in cancer clinical trials.

Address correspondence and reprint requests to: Joseph F. Costello, Ph.D., Brain Tumor Research Center, Department of Neurosurgery, Helen Diller Family Comprehensive Cancer Center, University of California San Francisco, San Francisco, CA, 94143. E-mail: jcostello@ cc.ucsf.edu.
Covalent modifications of DNA and amino acids on histones are two major mechanisms of epigenetic gene regulation. DNA methylation involves the addition of a methyl group to cytosine to create 5-methylcytosine. In mammals methylation occurs primarily at $5^{\prime}-\mathrm{CpG}-3^{\prime}$ dinucleotides, and occasionally at $\mathrm{CpNpGs}$ as well. ${ }^{1}$ DNA methylation is controlled by DNA methyltransferases (DNMT) that create (DNMT3A, DNMT3B) or maintain (DNMT1) patterns of methylation. ${ }^{2,3}$ DNA methylation is required for maintaining gene silencing on the inactive $\mathrm{X}$ chromosome, ${ }^{4-6}$ parental allele-specific expression of imprinted loci, ${ }^{7}$ and tissue and cell-typespecific gene expression. Methylation is also required for silencing transposable elements and maintaining genome stability ${ }^{8,9}$ and is a critical regulator of pluripotency genes. ${ }^{10-12}$

A second type epigenetic mechanism is the post-translational modification of $\mathrm{N}$-terminal tails of histone proteins by acetylation, methylation, phosphorylation, ubiquitylation, sumoylation, ADP ribosylation, biotinylation, and potentially other modifications. ${ }^{13}$ Like DNA methylation, specific enzymes also catalyze post-translational 
modifications of histones, and include acetyltransferases and deacetylases, methyltransferases and demethylases, among others. In contrast to DNA methylation, histone methylation can be mono-, di-, or tri-methylated on a single, specific lysine (i.e., at H3K4). Multiple types of modifications are present on a single histone molecule, increasing the combinatorial complexity, referred to as a "histone code." In addition to DNA methylation and histone modifications, there are other interrelated, potentially epigenetic mechanisms including specific deposition of histone variants, noncoding RNAs, chromatin remodeling, and nuclear organization of DNA. These mechanisms add additional layers of regulation and maintenance of gene expression states in both normal and diseased tissues.

\section{EPIGENETIC MECHANISMS REGULATING GENE EXPRESSION IN THE BRAIN}

Epigenetic mechanisms are critical to the development and function of the mammalian CNS). Global DNA methylation levels change during brain development, ${ }^{14}$ and methylation of specific genes can vary among different brain regions, cell types, and potentially even between the same cell type from different brain regions. ${ }^{15}$ The relationship between brain-region-specific gene expression $^{16}$ and brain-region-specific DNA methylation has not been fully explored. However, distinct CNS cell types may be differentially marked by DNA methylation. In murine astrogliogenesis, for example, DNA demethylation of the Gfap promoter including a critical STAT3 DNA binding site is associated with activation of Gfap transcription, a known marker of the astroglial lineage. ${ }^{17}$ One particular $\mathrm{CpG}$ site within the Gfap promoter is methylated in neural precursors and postmitotic neurons but unmethylated in astrocytes. ${ }^{17-19}$

DNA methyltransferases are important in CNS development and function. The maintenance methyltransferase DNMT1 is highly expressed in the mammalian brain, ${ }^{20-22}$ including in postmitotic neurons, despite its proposed primary role in copying methylation during DNA replication. DNA methylation changes in response to neural activity may be one function of DNMT1 in postmitotic neurons. ${ }^{23}$ Conditional Dnmtl deletion in murine postmitotic neurons does not affect overall DNA methylation levels or cell survival. ${ }^{24}$ On the other hand, conditional Dnmtl deletion in embryonic day 12 neuroblasts resulted in DNA hypomethylation and lethality immediately after birth due to CNS-associated respiratory failure, indicating a requirement for DNA methylation in these cells. Mosaic mice with 30\% Dnmt1-/cells survived into adulthood, but mutant cells were rapidly eliminated from the brain within 3 weeks of birth, further supporting the necessity of DNMT1 for CNS cell survival. Dnmt3b is expressed in the murine CNS for a short time during neurogenesis, whereas Dnmt3a is expressed in both prenatal and postnatal CNS. ${ }^{25}$ Dnmt $3 b$-/mice exhibit prenatal lethality and neural tube defects, demonstrating a critical role for DNMT3b in neurodevelopment. ${ }^{26}$ Mice with conditional deletion of Dnmt $3 a$ in the nervous system are apparently born healthy but die prematurely, displaying hypoactivity, abnormal walking, and poor performance on tests of neuromuscular function and motor coordination. ${ }^{27}$ Thus, the effects of Dnmt deficiency on brain functions are significant, but the specific effects on neurons and glia require further investigation.

Recent evidence indicates that neuronal differentiation is regulated in part by DNA demethylation and polycombmediated histone H3 K27 trimethylation (H3K27me3). ${ }^{28}$ DNA methylation contributes to repression of pluripotency in lineage-committed neural progenitors. Also, promoters marked by $\mathrm{H} 3 \mathrm{~K} 27 \mathrm{me} 3$ in neural stem cells often gain DNA methylation during differentiation. ${ }^{29}$ Thus, context-dependent interactions between different epigenetic mechanisms guide neural differentiation, and underscore the general importance of epigenetics in normal CNS development and maintenance of cellular identity.

Several human neurodevelopmental disorders are caused by mutations in genes encoding proteins involved in epigenetic mechanisms. Rett syndrome, for example, is a severe neurodevelopmental disorder caused by mutations in $M E C P 2$, which encodes a protein that can bind to methylated DNA and regulate gene expression. ${ }^{30} \mathrm{ICF}$ syndrome, which includes mental retardation, is caused by mutations in the de novo DNA methyltransferase DNMT3B. ${ }^{31-33}$ Furthermore, the dependence of the CNS on epigenetic regulation extends beyond DNA methylation and DNA methyltransferases, as mutations in genes encoding other epigenetic regulatory proteins can cause neurodevelopmental disorders. One example is JARIDI/ $S M C X$, encoding a JmjC-domain-containing histone demethylase, which, when mutated, causes a form of Xlinked mental retardation. ${ }^{34-36}$ These examples illustrate the importance of epigenetic control of gene expression in the development and function of the CNS. This knowledge of the critical role of epigenetic mechanisms and marks in the CNS provides a foundation for understanding the role of epigenetic regulation in tumors arising from CNS cells.

\section{DNA HYPOMETHYLATION AND CPG ISLAND HYPERMETHYLATION IN GLIOMAS}

Genome-wide or "global" hypomethylation occurs at a high frequency $(\sim 80 \%)$ in primary glioblastoma (GBM). ${ }^{37-39}$ The level of hypomethylation varies between tumors, ranging from near normal brain levels to approximately $50 \%$ of normal in approximately $20 \%$ of 
the cases, reflecting the massive demethylation of approximately 10 million $\mathrm{CpG}$ sites per tumor cell. Both single-copy loci and repetitive sequences can be hypomethylated in GBM. The most severe globally hypomethylated primary GBMs are also the most proliferative and are associated with demethylation and transcriptional activation of the putative oncogene MAGEA1. ${ }^{37,40}$ MAGEAl, a member of the MAGE family of genes, is one of a group of germline-specific genes that become transcriptionally activated in multiple types of human cancers, and this activation is correlated with genomewide hypomethylation and increased cellular proliferation. ${ }^{37,41}$ The expression of the protein products of these "cancer-testis antigen" genes in tumors results in the recognition of tumor-specific antigens on the cell surface by cytolytic $\mathrm{T}$ lymphocytes and also inhibits $\mathrm{p} 53$ function and response to chemotherapy in cancer cell lines. ${ }^{42,43}$ Interleukin-13Ra2 is another classic cancertestis antigen that is epigenetically activated in gliomas. ${ }^{44,45}$ IL13Ra2 expression is cancer cell-specific, and antibodies directed to it have been used to deliver toxic agents, such as Pseudomonas exotoxin to glioma cells in patients. Although the full consequences of genomic hypomethylation are unknown, it is sufficient to initiate tumorigenesis in mice, and can modulate tumor incidence in mouse models of cancer driven by genetic alterations..$^{8,46-48}$ The molecular consequences of global hypomethylation in gliomas have not been studied indepth.

Locus-specific hypermethylation, mostly at $\mathrm{CpG}$ island (CGI) promoters, is frequent in gliomas. CGIs are regions of $\sim 500 \mathrm{bp}-1 \mathrm{~kb}$ where $\mathrm{CpG}$ nucleotides are $\sim 5$ times more abundant compared with the rest of the genome. ${ }^{49}$ In gliomas, CGI promoter hypermethylation occurs at genes with diverse functions related to tumorigenesis and tumor progression, including cell-cycle regulation, DNA repair, apoptosis, angiogenesis, invasion, and drug resistance. For example, the retinoblastoma (RB), PI3K, and p53 pathways are affected by CGI promoter hypermethylation (including promoters of the genes CDKN2/p16, RB, PTEN, TP53 and p14 $\left.{ }^{A R F}\right) .{ }^{50-55}$ CGI hypermethylation in gliomas may also occur at genes that are not expressed in the brain, suggesting not all CGI methylation events are functionally important for tumorigenesis.

Screens for promoter hypermethylation in GBM have identified new tumor suppressor candidates, as well as bona fide tumor suppressors. One example is at chromosome $19 \mathrm{q}$, where deletions in gliomas and neuroblastomas have suggested the presence of a tumor suppressor gene. Expression array analysis of glioma cells treated with a DNA demethylating agent discovered a candidate tumor suppressor, epithelial membrane protein 3 (EMP3), a myelin-related gene involved in cell proliferation and cell-cell interactions. ${ }^{56}$ EMP3 is silenced by hypermethylation in primary gliomas and reintroduction of EMP3 in neuroblastoma cell lines with EMP3 silencing resulted in reduced colony formation in vitro and decreased xenograft growth in mice, suggesting tumor suppressor function. ${ }^{56}$

Promoter hypermethylation regulates the oncogenic and proliferation-promoting transforming growth factor (TGF)-beta signaling pathway in aggressive, highly proliferative GBMs. High levels of TGF-beta signaling are normally associated with poor prognosis. TGF-beta signaling promotes proliferation through the induction of platelet-derived growth factor $(P D G F)-B$. However, epigenetic silencing of $P D G F-B$ can override the increased proliferative effects of TGF-beta signaling. Specifically, $P D G F-B$ promoter hypermethylation prevents $P D G F-B$ transcriptional activation by TGF-beta-induced Smad proteins. ${ }^{57}$ The oncogenic affect of the TGF-beta pathway is therefore blocked by epigenetic alteration of one of its targets.

Genes involved in invasion and metastasis can also be affected by promoter hypermethylation in gliomas. A high frequency of astrocytomas (88\%), GBMs (87\%), and glioma cell lines (100\%) exhibit CGI promoter hypermethylation of the protocadherin-gamma subfamily A11 (PCDH-gamma-A11) gene, which is believed to be important in invasion of cancer cells into normal brain parenchyma. ${ }^{58}$ However, the use of the sensitive methylation-sensitive PCR method for detecting methylation may overestimate the percentage of tumors in which methylation reaches a biologically meaningful level.

Promoter hypermethylation can modulate sensitivity to drugs and radiotherapy in GBM. The best known example is $M G M T$ promoter methylation and response to DNA alkylating agents (see below), but there are other cases and this research area is likely to expand in the future. ${ }^{59,60}$ This suggests that epigenetic profiling might be one way to categorize GBMs and to rationally apply patient-specific therapy.

For other sets of genes, promoter DNA hypermethylation might not be causal or may not be required for gene silencing in cancer. For example, the enhancer of zeste 2 (EZH2) histone methyltransferase silences approximately $5 \%$ of gene promoters in prostate cancer cells by histone $\mathrm{H} 3$ lysine trimethylation independent of DNA methylation. ${ }^{61}$ The order and hierarchy of epigenetic modifications in the process of gene silencing is an area of extensive investigation will likely yield clues about potential primary causes of epigenetic silencing in GBM and other cancers.

\section{ABERRANT METHYLATION OF MGMT AND RESPONSE TO DNA ALKYLATING AGENTS}

One particularly important example of epigenetic silencing of a drug-resistance gene is promoter hypermeth- 
ylation of O6-methylguanine-DNA methyltransferase $(M G M T)$ in GBM. MGMT encodes a DNA repair protein that removes alkyl adducts at the O6 position of guanine and (less frequently) at the $\mathrm{O} 4$ position of thymine. ${ }^{62}$ Expression of MGMT protects normal cells from carcinogens; however, it can also protect cancer cells from chemotherapeutic alkylating agents. MGMT promoter methylation is negatively correlated with expression and is associated with decreased transcription factor binding in GBM cell lines. ${ }^{63,64}$ An initial report suggested a paradoxical positive correlation between MGMT methylation and expression in cancer cell lines. ${ }^{65}$ However, updates in the genomic structure of the MGMT gene showed that the paradoxically higher level of methylation in MGMT-expressing cells was located $50 \mathrm{~kb}$ downstream of the $M G M T$ promoter, well into the gene body. Hypermethylation of the MGMT promoter, leading to transcriptional silencing, occurs in multiple types of human cancers, including glioma, lymphoma, breast cancer, prostate cancer, and retinoblastoma. ${ }^{66}$ In human colorectal cancer, MGMT promoter methylation is associated with increased $G$ to $A$ transition mutations in TP53 and K-RAS. ${ }^{67,68}$ In GBM, MGMT promoter methylation correlates with mismatch repair deficiency and a putative hypermutator phenotype. ${ }^{69}$

$M G M T$ hypermethylation is associated with significantly longer survival in patients with GBMs and lowgrade gliomas treated with radiation and alkylating agents, including temozolomide, ${ }^{70,71}$ although it is unclear if this is directly due to reduced MGMT expression. Furthermore, MGMT hypermethylation is a predictor of the incidence and outcome of pseudoprogression, ${ }^{72}$ the progressive and enhancing lesions observed by MRI immediately after the end of treatment that are an effect of the radiotherapy and temozolomide treatment rather than tumor progression. ${ }^{72}$ MGMT hypermethylation is a late event in progressive oligodendrogliomas, suggesting that MGMT methylation may have a different prognostic value for oligodendrogliomas than for GBMs and lowgrade gliomas. ${ }^{73}$ The predictive value of MGMT methylation may be increased when used in conjunction with gene expression data. ${ }^{74}$ However, further technical refinement is needed for the commonly used MGMT methylation assay: particularly when applied to fixed tissue, the methylation-sensitive PCR method is prone to false positives. $^{75}$

\section{SUBSETS OF EPIGENETIC ALTERATIONS ARE TUMOR GRADE-SPECIFIC}

Different glioma subtypes and tumor grades display distinct aberrant DNA methylation profiles. ${ }^{76}$ Secondary GBMs have a higher overall frequency of promoter methylation compared to primary GBMs at least for the promoters of $p 14^{A R F}, p 16^{I N K 4 a}, R B 1, M G M T$, and TIMP-3. ${ }^{77}$
Low-grade gliomas and secondary GBMs show PTEN promoter methylation and activation of the PI3K pathway as measured by protein kinase $\mathrm{B}$ (PKB/AKT) phosphorylation, whereas PTEN promoter methylation is a rare occurrence in primary GBMs. ${ }^{78}$ On the other hand, the same epigenetic alterations can be shared by different types of brain tumors, even in different species. ${ }^{79} \mathrm{CNS}$ cancers other than gliomas also display distinct methylation profiles (e.g., $h M L H 1, T I M P 3, M G M T, p 73$, and THBS1 are frequently hypermethylated in schwannomas, ${ }^{80}$ and $N F 2$ is genetically and epigenetically altered in meningioma). ${ }^{81}$

The progression of glioma over time is associated with distinct epigenetic patterns. Malignant progression and shorter survival in astrocytoma are associated with p1 $4^{A R F}$ but not MGMT hypermethylation, suggesting that these are two distinct pathways in astrocytoma with different clinical consequences. ${ }^{82}$ Recurrence is also characterized by specific epigenetic marks: hypermethylation of the pro-apoptotic gene caspase- 8 is frequently associated with relapsed GBM. ${ }^{83}$ Increased recurrence-free survival is associated with CITED4 hypermethylation at $1 \mathrm{p} 43.2$ and $1 \mathrm{p}$ and $19 \mathrm{q}$ losses. ${ }^{84}$ Murine glioma models also provide evidence of a role for epigenetic mechanisms in modifying tumor progression. In a mouse model of astrocytoma with mutant $\operatorname{Trp} 53$ and $N f 1$, a genetic modifier controlling susceptibility to progression is itself epigenetically modified. ${ }^{85}$

\section{EPIGENETIC ALTERATIONS IN GLIOMA TUMOR-INITIATING CELLS}

DNA hypermethylation can alter the differentiation properties of the fraction of glioma cells believed to be putative cancer stem cells, also called tumor-initiating cells. Normal astroglial differentiation is regulated by the bone morphogenetic protein (BMP)-mediated and ciliary neurotrophic factor-mediated Jak/STAT pathway. In a subset of GBM tumor-initiating cells, this differentiation pathway is inhibited by epigenetic silencing of the BMP receptor 1B (BMPR1B) gene. ${ }^{86}$ This silencing is dependent on the histone methyltransferase enhancer of zeste 2 (EZH2) and DNA methylation, and could be reversed by treatment with a DNA demethylating agent. These data demonstrate that a subset of human GBM cells are blocked from differentiation though a defect in the BMP signaling pathway. Furthermore, forced expression of $B M P R I B$ could restore the differentiation potential of these cells and can decrease tumorigenicity. These findings reinforce the idea that future epigenetic therapies might be applied rationally, guided by patient-specific epigenetic profiling.

The gene encoding the CD133 cell-surface marker used to identify and enrich GBM tumor-initiating cells is itself subject to epigenetic regulation. In GBM cell lines, 
CD133 promoter CGI DNA methylation was negatively correlated with CD133 expression. ${ }^{87}$ In pure CD133+ GBM lines, this methylation was absent. Furthermore, CD133 methylation was not observed in normal brain but only in cultured and primary tumors, suggesting that it could be a tumor-specific epigenetic marker. In contrast, another study found CD133 promoter CGI methylation in brain and hypomethylation in GBMs with high expression of aberrant of $C D 133$ transcripts. ${ }^{88}$ Follow-up studies are needed to more thoroughly appreciate the functional importance of these results.

\section{MAPPING GENOME-WIDE METHYLATION PATTERNS IN BRAIN TUMORS}

There are multiple strategies for discovering novel epigenetic alterations in brain tumors. One approach is to examine candidate loci that are affected by deletion or mutation on one allele, based on the idea that DNA hypermethylation might inactivate the other allele, leading to complete silencing or loss of normal function. This candidate gene strategy is more likely to discover hypermethylation of tumor suppressors rather than hypomethylation events. A more unbiased approach is to scan the entire genome for both hypermethylation and hypomethylation events. For gliomas, this was approached first through the use of methyl-sensitive restriction enzymes using restriction landmark genomic scanning. ${ }^{89-94}$ Newer methods such as reduced representation bisulfite sequencing ${ }^{95}$ and methylated DNA immunoprecipitation combined with DNA microarrays ${ }^{96}$ or massively parallel sequencing ${ }^{97}$ more completely interrogate the epigenome and are poised to dramatically augment our understanding of epigenetic dysregulation in gliomas and other cancers.

An additional approach to uncovering epigenetically altered genes is to treat cells with the DNMT1 inhibitor 5-aza-2'-deoxycytidine (5-aza) and/or the histone deacetylase inhibitor trichostatin A (TSA) followed by gene expression microarray analysis. ${ }^{98}$ This strategy identified brain expressed X-linked 1 (BEXI) and 2 $(B E X 2)$ as candidate tumor suppressors in glioma ${ }^{99}$ and SPINT2 in medulloblastoma. ${ }^{100}$ Another study identified $>160$ genes upregulated by combined 5-aza and TSA treatment in glioma cell lines and provided evidence that several of the epigenetically silenced loci are aberrantly methylated by the combined action of DNMT1 and DNMT3B, whereas others are regulated only by a single DNMT. ${ }^{101} 5$-aza treatment of short-term cultured primary GBMs identified two genes, runt-related transcription factor 3 (RUNX3) and testin (TES), with aberrant methylation. ${ }^{102}$ In this chemical approach, numerous genes may be indirectly activated. Thus, follow-up analyses of the epigenetic marks at the activated genes in primary tumors are essential.

\section{ALTERATIONS IN HISTONE MODIFICATIONS IN GLIOMA}

In addition to DNA hypermethylation, cancer genes can be silenced or activated by aberrant patterns of histone modifications, either alone, or in combination with DNA methylation. Silenced CGI promoters, for example, are characterized by increased histone $\mathrm{H} 3 \mathrm{~K} 9$ methylation and loss of H3K9 acetylation. In embryonic stem cells, the dual presence of inactivating $\mathrm{H} 3 \mathrm{~K} 27$ methylation and activation-associated $\mathrm{H} 3 \mathrm{~K} 4$ methylation, called bivalent domains, is believed to create a "poised" chromatin state for developmentally regulated genes, allowing silencing in embryonic stem cells and subsequent transcriptional activation or repression in differentiated cells. ${ }^{103}$ Bivalent domains, along with additional repressive marks (dimethylated H3K9 and trimethylated H3K9), are found in embryonal carcinoma cells at genes that are frequently silenced by DNA hypermethylation in adult human cancer cells. These histone modifications are hypothesized to predispose tumor suppressor genes to DNA hypermethylation and heritable gene silencing. ${ }^{104}$ Trimethylation of H3K27 marks silenced genes in the absence of DNA hypermethylation, although this has not yet been shown in gliomas.

Genes encoding DNMTs are not frequently mutated in human cancers, with one report of DNMT1 mutations in colorectal cancer. ${ }^{105}$ However, overexpression of DNMT1, 3a, and $3 \mathrm{~b}$ has been reported in multiple tumor types. ${ }^{106}$ Furthermore, there are many instances of genetic alterations and/or deregulated expression of genes encoding histone-modifying enzymes. In acute leukemias for example, translocations involving the mixed lineage leukemia $(M L L)$ gene, encoding an $\mathrm{H} 3 \mathrm{~K} 4$ methyltransferase, are common. ${ }^{107}$ These translocations result in MLL fusion proteins that have lost $\mathrm{H} 3 \mathrm{~K} 4$ methyltransferase activity. The polycomb protein EZH2, an H3K27 methyltransferase, is overexpressed and amplified in multiple types of human cancers. ${ }^{108}$

The gene encoding BMI-1, a member of the polycomb group complex that regulates histone H3K27 methylation, is subject to frequent copy number alterations in both low-grade and high-grade gliomas, and BMI- 1 deletions are associated with poor prognosis in patients. ${ }^{109}$ Furthermore, BMI-1 is also upregulated in a proportion of gliomas. ${ }^{110}$ An important target of BMI-1 transcriptional repression is the Ink4a/Arf locus, harboring two tumor suppressors in gliomas. Repression of this locus by BMI- 1 promotes cell proliferation. ${ }^{111,112}$ However, in an orthotopic transplantation model, BMI-1 is also required for tumorigenesis independent of Ink4a/Arf, suggesting additional targets of epigenetic regulation by BMI-1 may be important in glial tumorigenesis. ${ }^{113,114}$

Mutations resulting in altered histone acetyltransferase activity also occur in cancer-related diseases: $C B P \mathrm{mu}-$ 
tations, abolishing histone acetyltransferase activity, cause Rubenstein-Taybi syndrome, a developmental disorder that is associated with a higher risk of cancer. ${ }^{115} \mathrm{In}$ GBM, there is preliminary evidence for deregulation of genes controlling histone modifications. Expression of some histone deacetylase (HDAC) proteins is reported to be altered in GBM. Class II and IV HDACs displayed decreased mRNA expression in GBMs compared to lowgrade astrocytomas and normal brain, and overall histone H3 was more acetylated in GBMs. ${ }^{116}$ Large-scale sequencing of protein-coding genes in GBMs uncovered mutations in many genes involved in epigenetic regulation, including histone deacetylases $H D A C 2$ and $H D A C 9$, histone demethylases $J M J D 1 A$ and $J M J D 1 B$, histone methyltransferases SET7, SETD7, MLL, MLLA and methyl-CpG binding domain protein $1(M B D 1) .{ }^{117}$ These intriguing initial studies suggest that alterations in epigenetic mechanisms could be a major defect in GBM.

\section{WHAT CAUSES EPIGENETIC ALTERATIONS IN GLIOMA?}

The cause(s) and consequences of epigenetic dysregulation in GBM, and cancer in general, remain an area of intense investigation, with some progress being made in understanding the consequences. The causes, however, remain mostly obscure. Multiple causes have been proposed for aberrant DNA methylation. Alteration in DNA methyltransferase enzymes is a possibility, and aberrant expression of DNMTs has been observed. For example, $D N M T 3 b$ splice variants and aberrant transcripts are overexpressed or ectopically expressed in some cancers and cancer cell lines. The splice variant DNMT3b4, which lacks the conserved methyltransferase motifs IX and $\mathrm{X}$, is overexpressed in hepatocellular carcinoma. ${ }^{118}$ Furthermore, its forced overexpression caused pericentromeric hypomethylation, suggesting that DNMT3b4 might act in a dominant negative manner, blocking methylation of this region of repetitive DNA. Another study of primary acute leukemia cells and cancer cell lines (including GBM cell lines) identified expression of over 20 aberrant DNMT3b transcripts with altered 5' splicing, coding for truncated proteins that lack the $\mathrm{C}$-terminal catalytic domain. ${ }^{119}$ Although aberrant DNMT3b transcripts account for only a small fraction of total DNMT3b mRNA in this study, at least some of these are translated to proteins, and their overexpression was linked to changes in DNA methylation level and mRNA abundance of other genes. Of these transcripts, DNMT3b4 is the only one shown to produce a dominant negativeacting protein in cancer cells, leading to hypomethylation. Increased DNMT1 and decreased DNMT3a expression have also been reported in GBM cell lines, and the decrease in DNMT3a is hypothesized to contribute to
Sat2 hypomethylation in the tumor stem cell subpopulation. ${ }^{39}$

In theory, either an initial genetic or epigenetic abnormality could result in tumorigenesis and cause further downstream genetic and epigenetic changes. In glioma, epigenetic silencing of the tumor suppressor gene WNK lysine deficient protein kinase 2 (WNK2) was significantly associated with combined deletion of chromosomes $1 \mathrm{p}$ and 19q. ${ }^{120}$ WNK2 indirectly inhibits MEK1, enhancing growth promoting signals through EGFR, which is often itself overexpressed and constitutively active due to genomic amplification and rearrangement, respectively. ${ }^{121,122}$ Thus, it is possible that the epigenetic silencing of WNK2 interacts on a functional level with genetic alteration of EGFR signaling, a common abnormality in GBM. A GBM with a point mutation in $W N K 2$ was reported recently, ${ }^{117}$ whereas commonly occurring cancers exhibit relatively frequent point mutations in all four $W N K$ genes. In meningioma, grade-specific epigenetic alterations in $W N K 2$ are the dominant mechanism of gene inactivation, occurring in $83 \%$ and $71 \%$ of grade II and III tumors. ${ }^{123}$

\section{EPIGENETIC THERAPIES FOR GLIOMA}

Epigenetic-based therapy such as the DNMT inhibitor decitabine (5-aza-2'-deoxycytidine) and the HDAC inhibitor (HDACi) suberoylanilide hydroxamic acid (SAHA; vorinostat) are currently being tested in multiple cancers, although only HDACis are in trials for GBM treatments. In contrast to genetic mutations, which are "hard-wired" once, mutated epimutations, such as promoter hypermethylation and histone acetylation status are theoretically reversible by drug treatment or possibly diets.

A major unresolved issue for epigenetic therapy of cancer is target specificity. First, some genes that require DNA methylation or histone deacetylation for silencing in normal cells could be unintentionally activated by agents that inhibit DNMTs or HDACs. Second, cancer genomes are characterized by both DNA hypermethylation and hypomethylation. Therefore, using drugs that reactivate silenced tumor suppressors may have the undesired effect of further activating oncogenes through hypomethylation. These problems should be addressed to gain a more complete understanding of the molecular events resulting from epigenetic-based therapy.

HDACs catalyze the deacetylation of lysine residues within the N-terminal tails of core histones and also within nonhistone proteins. As a result, their effects are complex and involve histone and nonhistone substrates, and the mechanism of specificity for each HDAC is not fully understood. In general, HDACs promote a closed chromatin structure that represses transcription. There are 18 known HDACs in humans, divided into five main 
classes, with different target specificities. ${ }^{124}$ HDACs of class I (HDACs 1, 2, 3 and 8), class IIA (HDACs 4, 5, 7, and 9), class IIB (HDACs 6 and 10), and class IV (HDAC 11) all contain zinc in their active sites and are inhibited by the HDACis TSA and SAHA. Class III HDACs (sirtuins) do not contain zinc and are not inhibited by TSA or SAHA.

The rationale for using HDACis in cancer therapy is two-fold. First, HDACis promote a more open chromatin conformation and might therefore permit better access of DNA damaging agents to the chromatin and increase the sensitivity to killing by these agents. Second, HDACis will reverse some of the aberrant epigenetic gene silencing in GBMs, presumably leading to enhanced cell-cycle arrest and apoptosis from DNA-damaging agents. ${ }^{125} \mathrm{Al}-$ though it is not clear how consistently HDACis activate specific pathways from one GBM to the next, HDACis do synergize with DNA damaging agents in arresting or killing glioma cells in vitro.

HDACis are comprised of several classes of compounds, including hydroxymates (SAHA, TSA), cyclic peptides (depsipeptide), aliphatic acids (valproic acid, butyrate), and benzamides. No single HDACi is effective against all HDACs. HDACis cause increased acetylation of histone and nonhistone proteins, and can reactivate $p 21$, which contributes to cell-cycle arrest. ${ }^{126,127}$ Noncancerous cells are more resistant to the effects of HDACi, but the reasons for this selective sensitivity are unclear. ${ }^{128}$ HDACis alter the expression levels of only a subset of expressed genes in transformed cells $(\sim 2$ to $10 \%)$, and both increases and decreases in transcript levels are observed. ${ }^{129-132}$

SAHA is currently being tested as a monotherapy or in combination therapy in 5 phase I or I-II clinical trials for gliomas. SAHA targets class I and II HDACs at micromolar concentrations, and preclinical studies found that it sensitizes glioma cells in vitro, ex vivo, and in vivo to chemotherapy and radiation. ${ }^{133-137}$ SAHA treatment increased $p 21$ promoter histone $\mathrm{H} 3$ acetylation in the U87 glioma cell line, and inhibited the proliferation of GL26 glioma cells implanted in mice. ${ }^{126}$ Two of the current clinical trials test SAHA in combination with temozolomide, and one also includes radiotherapy. A third trial consists of SAHA plus isotretinoin and carboplatin, a fourth uses SAHA plus bortezomib, and a fifth is testing SAHA as a monotherapy.

Trials are also currently underway for two additional HDACis, valproic acid (Depakene; Depakote) and depsipeptide (Romedepsin; FK-228), and there are additional HDACi compounds that are not yet in clinical trials. Valproic acid is being tested against GBM in combination with temozolomide plus radiation, and in a broader second trial against neuronal tumors and brain metastases in combination with etoposide. Depsipeptide monotherapy is being tested against high-grade gliomas.
Valproic acid is active against class I and IIA HDACs at millimolar concentrations; depsipeptide is active against class I HDACs at nanomolar concentrations. An additional HDACi not yet in clinical trials is pivaloyloxymethyl butyrate (AN-9), a derivative of butyrate. Butyrate is an aliphatic acid HDACi effective against class I and IIA HDACs at millimolar concentrations. AN-9 shows efficacy in GBM cell culture and animal models. ${ }^{138,139}$ Furthermore, AN-9 sensitized GBM mouse xenografts to radiation and showed decreased tumor growth and increased survival. There are several HDACis that have shown efficacy against cancer cells but have not yet been tested for gliomas including panobinostat (LBH589) ${ }^{140}$ and belinostat (PXD101). ${ }^{141}$ The discovery and development of new epigenetic enzyme-targeting compounds is an area of active research in the pharmaceutical industry.

\section{MOLECULAR BIOMARKERS OF RESPONSE AND PATIENT SURVIVAL}

Blood plasma in cancer patients contains DNA derived from tumor cells due to necrotic or apoptotic cancer cells releasing genomic DNA. This potentially provides a less invasive method for biomarker detection. Aberrantly hypermethylated cancer genes found in plasma could be one such type of biomarker. This type of analysis may provide clinically useful information relating to diagnosis, prognosis, and follow-up after therapy. Methylated tumor-specific biomarkers have, in fact, been found in plasma and serum for several types of cancer. ${ }^{142,143}$ There is a significant amount of tumor DNA in the plasma of high-grade glioma patients, and in $60 \%$ of patients, the same methylated promoters (p16INK4a, $M G M T, p 73$, and RARbeta were tested) could be detected in both tumor and plasma DNA. ${ }^{144}$ The frequency of detection of methylated promoters in plasma of GBM patients was similar to that in other cancers, suggesting that the blood-brain barrier does not completely block the transfer of tumor DNA to plasma. This method is theoretically highly sensitive, because small amounts of methylated DNA can be detected in a sample by methylation-sensitive PCR.

\section{FUTURE DIRECTIONS}

Epigenetic studies of glioma are poised to 1) make substantial contributions to the understanding of glioma biology, 2) identify new predictive biomarkers, and 3) discover novel targets for therapy. New models, such as patient GBM-derived tumor stem cells grown in neurosphere culture may be a valuable addition to epigenetic research into GBM, particularly if the epigenetic profiles of the corresponding primary tumors are retained, as has been shown for gene expression patterns and invasive growth patterns of these cells. ${ }^{145,146}$ Epigenomic profil- 
ing of DNA methylation, histone modifications, and noncoding RNAs (such as microRNAs) in primary tumors, orthotopic xenografts, and tumor neurospheres are strategies that will likely uncover many additional epigenetic alterations in gliomas, and therefore targets for therapy.

There are still many questions remaining about the role of epigenetics in gliomas. The causes and consequences of epigenetic alterations are still mostly unknown, and the relative contributions of genetic and environmental factors in causing epigenetic alterations have not been quantified. Why are some genes or pathways more affected by epigenetic rather than genetic alterations or vice versa? It is clear that approaches simultaneously examining both genetic and epigenetic defects, complemented with functional studies, will be essential. It will also be important to understand the effects of HDACi on the entire cancer acetylome to elucidate the molecular consequences of this treatment strategy.

Combination therapy with both DNMT and HDACis might be one strategy against glioma. A dual treatment approach may have a synergistic effect on gene activation, and could allow lower doses of each drug to be used. Such a strategy is being tested in a clinical trial for myelodysplastic syndrome and acute myelogenous leukemia using the DNMT inhibitor decitabine with or without valproic acid (clinicaltrials.gov ID NCT00414310).

An area that is mostly unexplored in glioma is the development and testing of drugs directed against histone modifications other than acetylation. H3K27 methylation at silenced tumor suppressor promoters could be targeted to reactivate these genes, for example using the S-adenosylhomocysteine hydrolase inhibitor 3-deazaneplanocin A. ${ }^{147}$ As epigenetic modifications are better understood and more types are discovered, additional epigenetic drug targets can be tested in GBMs and other cancers.

Consortiums using high-throughput genomic and epigenomic approaches are helping to unravel the genetic and epigenetic alterations that contribute to GBM. The Cancer Genome Atlas (TCGA; http://cancergenome. nih.gov/about/mission.asp) is examining genomic changes and DNA methylation in three cancers, including GBM $^{69}{ }^{6}$ A complementary, privately funded project sequenced the exons of 20,661 protein coding genes in GBM, identifying recurrent mutations in the active site of isocitrate dehydrogenase $1(I D H 1)$ in $12 \%$ of GBM patients, ${ }^{117}$ and 70 to $80 \%$ of low-grade glioma patients. The International Cancer Genome Consortium is an additional large-scale effort, which will include genomic and epigenomic profiling. Considering there are 30,000 CGIs in the human genome, tiling microarrays or nextgeneration sequencing methods may be required to fully investigate aberrant CGI DNA methylation in CNS tumors. A full genome-scale map of DNA methylation and histone modifications in glioma will be a key reference for accelerating understanding of epigenetic mechanisms underlying this deadly disease.

Acknowledgements: This work was supported in part by the National Brain Tumor Society, Accelerated Brain Cancer Cure, Goldhirsh Foundation and the Karen Osney Brownstein Endowed Chair to R.P.N., and by a postdoctoral T32 NRSA (NIH Institutional Research Service Award in Molecular and Cellular Mechanisms in Cancer) to J.F.C.

\section{REFERENCES}

1. Clark SJ, Harrison J, Frommer M. CpNpG methylation in mammalian cells. Nat Genet 1995;10:20-27.

2. Bestor TH. Cloning of a mammalian DNA methyltransferase. Gene 1988;74:9-12.

3. Okano M, Xie S, Li E. Cloning and characterization of a family of novel mammalian DNA (cytosine-5) methyltransferases. Nat Genet 1998;19:219-220.

4. Mohandas T, Sparkes RS, Shapiro LJ. Reactivation of an inactive human $\mathrm{X}$ chromosome: evidence for $\mathrm{X}$ inactivation by DNA methylation. Science 1981;211:393-396.

5. Wolf SF, Jolly DJ, Lunnen KD, Friedmann T, Migeon BR. Methylation of the hypoxanthine phosphoribosyltransferase locus on the human X chromosome: implications for X-chromosome inactivation. Proc Natl Acad Sci U S A 1984;81:2806-2810.

6. Hansen RS, Gartler SM. 5-Azacytidine-induced reactivation of the human X chromosome-linked PGK1 gene is associated with a large region of cytosine demethylation in the $5^{\prime} \mathrm{CpG}$ island. Proc Natl Acad Sci U S A 1990;87:4174-4178.

7. Li E, Beard C, Jaenisch R. Role for DNA methylation in genomic imprinting. Nature 1993;366:362-365.

8. Eden A, Gaudet F, Waghmare A, Jaenisch R. Chromosomal instability and tumors promoted by DNA hypomethylation. Science 2003;300:455.

9. Walsh CP, Chaillet JR, Bestor TH. Transcription of IAP endogenous retroviruses is constrained by cytosine methylation. Nat Genet 1998;20:116-117.

10. Fouse SD, Shen Y, Pellegrini M, et al. Promoter CpG methylation contributes to ES cell gene regulation in parallel with Oct4/ Nanog, PcG complex, and histone H3 K4/K27 trimethylation. Cell Stem Cell 2008;2:160-169.

11. Mikkelsen TS, Hanna J, Zhang X, et al. Dissecting direct reprogramming through integrative genomic analysis. Nature 2008; 454:49-55.

12. Takahashi K, Yamanaka S. Induction of pluripotent stem cells from mouse embryonic and adult fibroblast cultures by defined factors. Cell 2006;126:663-676.

13. Turner BM. Reading signals on the nucleosome with a new nomenclature for modified histones. Nat Struct Mol Biol 2005; 12:110-112.

14. Tawa R, Ono T, Kurishita A, Okada S, Hirose S. Changes of DNA methylation level during pre- and postnatal periods in mice. Differentiation 1990;45:44-48.

15. Ladd-Acosta C, Pevsner J, Sabunciyan S, et al. DNA methylation signatures within the human brain. Am J Hum Genet 2007;81: 1304-1315.

16. Lein ES, Hawrylycz MJ, Ao N, et al. Genome-wide atlas of gene expression in the adult mouse brain. Nature 2007;445:168-176.

17. Takizawa T, Nakashima K, Namihira M, et al. DNA methylation is a critical cell-intrinsic determinant of astrocyte differentiation in the fetal brain. Dev Cell 2001;1:749-758.

18. Condorelli DF, Dell'Albani P, Conticello SG, et al. A neuralspecific hypomethylated domain in the $5^{\prime}$ flanking region of the glial fibrillary acidic protein gene. Dev Neurosci 1997;19:446456.

19. Condorelli DF, Nicoletti VG, Barresi V, et al. Tissue-specific DNA methylation patterns of the rat glial fibrillary acidic protein gene. J Neurosci Res 1994;39:694-707. 
20. Goto K, Numata M, Komura JI, et al. Expression of DNA methyltransferase gene in mature and immature neurons as well as proliferating cells in mice. Differentiation 1994;56:39-44.

21. Inano K, Suetake I, Ueda T, et al. Maintenance-type DNA methyltransferase is highly expressed in post-mitotic neurons and localized in the cytoplasmic compartment. J Biochem (Tokyo) 2000;128:315-321.

22. Trasler JM, Trasler DG, Bestor TH, Li E, Ghibu F. DNA methyltransferase in normal and Dnmtn/Dnmtn mouse embryos. Dev Dyn 1996;206:239-247.

23. Martinowich $\mathrm{K}$, Hattori D, Wu H, et al. DNA methylation-related chromatin remodeling in activity-dependent BDNF gene regulation. Science 2003;302:890-893.

24. Fan G, Beard C, Chen RZ, et al. DNA hypomethylation perturbs the function and survival of CNS neurons in postnatal animals. J Neurosci 2001;21:788-797.

25. Feng J, Chang H, Li E, Fan G. Dynamic expression of de novo DNA methyltransferases Dnmt3a and Dnmt3b in the central nervous system. J Neurosci Res 2005;79:734-746.

26. Okano M, Bell DW, Haber DA, Li E. DNA methyltransferases Dnmt3a and Dnmt3b are essential for de novo methylation and mammalian development. Cell 1999;99:247-257.

27. Nguyen S, Meletis K, Fu D, Jhaveri S, Jaenisch R. Ablation of de novo DNA methyltransferase Dnmt3a in the nervous system leads to neuromuscular defects and shortened lifespan. Dev Dyn 2007; 236:1663-1676.

28. Ma DK, Jang MH, Guo JU, et al. Neuronal Activity-Induced Gadd45b Promotes Epigenetic DNA Demethylation and Adult Neurogenesis. Science 2009.

29. Mohn F, Weber M, Rebhan M, et al. Lineage-specific polycomb targets and de novo DNA methylation define restriction and potential of neuronal progenitors. Mol Cell 2008;30:755-766.

30. Amir RE, Van den Veyver IB, Wan M, et al. Rett syndrome is caused by mutations in X-linked MECP2, encoding methyl-CpGbinding protein 2. Nat Genet 1999;23:185-188.

31. Hansen RS, Wijmenga C, Luo P, et al. The DNMT3B DNA methyltransferase gene is mutated in the ICF immunodeficiency syndrome. Proc Natl Acad Sci U S A 1999;96:14412-14417.

32. Xu GL, Bestor TH, Bourc'his D, et al. Chromosome instability and immunodeficiency syndrome caused by mutations in a DNA methyltransferase gene. Nature 1999;402:187-191.

33. Tuck-Muller CM, Narayan A, Tsien F, et al. DNA hypomethylation and unusual chromosome instability in cell lines from ICF syndrome patients. Cytogenet Cell Genet 2000;89:121-128.

34. Jensen LR, Amende M, Gurok U, et al. Mutations in the JARID1C gene, which is involved in transcriptional regulation and chromatin remodeling, cause $\mathrm{X}$-linked mental retardation. Am J Hum Genet 2005;76:227-236.

35. Tahiliani M, Mei P, Fang R, et al. The histone H3K4 demethylase SMCX links REST target genes to X-linked mental retardation. Nature 2007;447:601-605.

36. Akbarian S. Diseases of the mind and brain: Rett's syndrome. Am J Psychiatry 2002;159:1103.

37. Cadieux B, Ching TT, VandenBerg SR, Costello JF. Genomewide hypomethylation in human glioblastomas associated with specific copy number alteration, methylenetetrahydrofolate reductase allele status, and increased proliferation. Cancer Res 2006;66:8469-8476.

38. Gama-Sosa MA, Slagel VA, Trewyn RW, et al. The 5-methylcytosine content of DNA from human tumors. Nucleic Acids Res 1983;11:6883-6894.

39. Fanelli M, Caprodossi S, Ricci-Vitiani L, et al. Loss of pericentromeric DNA methylation pattern in human glioblastoma is associated with altered DNA methyltransferases expression and involves the stem cell compartment. Oncogene 2008;27:358365.

40. Yu J, Zhang H, Gu J, et al. Methylation profiles of thirty four promoter-CpG islands and concordant methylation behaviours of sixteen genes that may contribute to carcinogenesis of astrocytoma. BMC Cancer 2004;4:65.

41. De Smet C, De Backer O, Faraoni I, et al. The activation of human gene MAGE-1 in tumor cells is correlated with genome- wide demethylation. Proc Natl Acad Sci U S A 1996;93: $7149-7153$.

42. Van Der Bruggen P, Zhang Y, Chaux P, et al. Tumor-specific shared antigenic peptides recognized by human $\mathrm{T}$ cells. Immunol Rev 2002;188:51-64.

43. Liu G, Ying H, Zeng G, et al. HER-2, gp100, and MAGE-1 are expressed in human glioblastoma and recognized by cytotoxic $\mathrm{T}$ cells. Cancer Res 2004;64:4980-4986.

44. Debinski W, Obiri NI, Powers SK, Pastan I, Puri RK. Human glioma cells overexpress receptors for interleukin 13 and are extremely sensitive to a novel chimeric protein composed of interleukin 13 and pseudomonas exotoxin. Clin Cancer Res 1995; 1:1253-1258.

45. Debinski W, Gibo DM. Molecular expression analysis of restrictive receptor for interleukin 13, a brain tumor-associated cancer/ testis antigen. Mol Med 2000;6:440-449.

46. Gaudet F, Hodgson JG, Eden A, et al. Induction of tumors in mice by genomic hypomethylation. Science 2003;300:489-492.

47. Howard G, Eiges R, Gaudet F, Jaenisch R, Eden A. Activation and transposition of endogenous retroviral elements in hypomethylation induced tumors in mice. Oncogene 2008;27:404-408.

48. Holm TM, Jackson-Grusby L, Brambrink T, et al. Global loss of imprinting leads to widespread tumorigenesis in adult mice. Cancer Cell 2005;8:275-285.

49. McClelland M, Ivarie R. Asymmetrical distribution of CpG in an "average" mammalian gene. Nucleic Acids Res 1982;10:78657877.

50. Costello JF, Berger MS, Huang HS, Cavenee WK. Silencing of p16/CDKN2 expression in human gliomas by methylation and chromatin condensation. Cancer Res 1996;56:2405-2410.

51. Baeza N, Weller M, Yonekawa Y, Kleihues P, Ohgaki H. PTEN methylation and expression in glioblastomas. Acta Neuropathol 2003;106:479-485.

52. Nakamura M, Yonekawa Y, Kleihues P, Ohgaki H. Promoter hypermethylation of the RB1 gene in glioblastomas. Lab Invest 2001;81:77-82.

53. Amatya VJ, Naumann U, Weller M, Ohgaki H. TP53 promoter methylation in human gliomas. Acta Neuropathol 2005;110:178184.

54. Watanabe T, Yokoo H, Yokoo M, et al. Concurrent inactivation of RB1 and TP53 pathways in anaplastic oligodendrogliomas. J Neuropathol Exp Neurol 2001;60:1181-1189.

55. Bello MJ, Rey JA. The p53/Mdm2/p14ARF cell cycle control pathway genes may be inactivated by genetic and epigenetic mechanisms in gliomas. Cancer Genet Cytogenet 2006;164:172 173.

56. Alaminos M, Davalos V, Ropero S, et al. EMP3, a myelin-related gene located in the critical $19 q 13.3$ region, is epigenetically silenced and exhibits features of a candidate tumor suppressor in glioma and neuroblastoma. Cancer Res 2005;65:2565-2571.

57. Bruna A, Darken RS, Rojo F, et al. High TGFbeta-Smad activity confers poor prognosis in glioma patients and promotes cell proliferation depending on the methylation of the PDGF-B gene. Cancer Cell 2007;11:147-160.

58. Waha A, Guntner S, Huang TH, et al. Epigenetic silencing of the protocadherin family member PCDH-gamma-A11 in astrocytomas. Neoplasia 2005;7:193-199.

59. Zhou H, Miki R, Eeva M, et al. Reciprocal regulation of SOCS 1 and SOCS3 enhances resistance to ionizing radiation in glioblastoma multiforme. Clin Cancer Res 2007;13:2344-2353.

60. Zardo G, Tiirikainen MI, Hong C, et al. Integrated genomic and epigenomic analyses pinpoint biallelic gene inactivation in tumors. Nat Genet 2002;32:453-458.

61. Kondo Y, Shen L, Cheng AS, et al. Gene silencing in cancer by histone $\mathrm{H} 3$ lysine 27 trimethylation independent of promoter DNA methylation. Nat Genet 2008;40:741-750.

62. Gerson SL. MGMT: its role in cancer aetiology and cancer therapeutics. Nat Rev Cancer 2004;4:296-307.

63. Costello JF, Futscher BW, Kroes RA, Pieper RO. Methylationrelated chromatin structure is associated with exclusion of transcription factors from and suppressed expression of the O-6methylguanine DNA methyltransferase gene in human glioma cell lines. Mol Cell Biol 1994;14:6515-6521. 
64. Costello JF, Futscher BW, Tano K, Graunke DM, Pieper RO. Graded methylation in the promoter and body of the O6-methylguanine DNA methyltransferase (MGMT) gene correlates with MGMT expression in human glioma cells. J Biol Chem 1994; 269:17228-17237.

65. Pieper RO, Costello JF, Kroes RA, et al. Direct correlation between methylation status and expression of the human O-6-methylguanine DNA methyltransferase gene. Cancer Commun 1991; 3:241-253.

66. Esteller M, Hamilton SR, Burger PC, Baylin SB, Herman JG. Inactivation of the DNA repair gene O6-methylguanine-DNA methyltransferase by promoter hypermethylation is a common event in primary human neoplasia. Cancer Res 1999;59:793-797.

67. Esteller M, Risques RA, Toyota M, et al. Promoter hypermethylation of the DNA repair gene $\mathrm{O}(6)$-methylguanine-DNA methyltransferase is associated with the presence of G:C to A:T transition mutations in p53 in human colorectal tumorigenesis. Cancer Res 2001;61:4689-4692.

68. Esteller M, Toyota M, Sanchez-Cespedes M, et al. Inactivation of the DNA repair gene O6-methylguanine-DNA methyltransferase by promoter hypermethylation is associated with $\mathrm{G}$ to A mutations in K-ras in colorectal tumorigenesis. Cancer Res 2000;60: $2368-2371$.

69. McLendon R, Friedman A, Bigner D, et al. Comprehensive genomic characterization defines human glioblastoma genes and core pathways. Nature 2008.

70. Hegi ME, Diserens AC, Gorlia T, et al. MGMT gene silencing and benefit from temozolomide in glioblastoma. N Engl J Med 2005;352:997-1003.

71. Everhard S, Kaloshi G, Criniere E, et al. MGMT methylation: a marker of response to temozolomide in low-grade gliomas. Ann Neurol 2006;60:740-743.

72. Brandes AA, Franceschi E, Tosoni A, et al. MGMT promoter methylation status can predict the incidence and outcome of pseudoprogression after concomitant radiochemotherapy in newly diagnosed glioblastoma patients. J Clin Oncol 2008;26: 2192-2197.

73. Lavon I, Zrihan D, Zelikovitch B, et al. Longitudinal assessment of genetic and epigenetic markers in oligodendrogliomas. Clin Cancer Res 2007;13:1429-1437.

74. Murat A, Migliavacca E, Gorlia T, et al. Stem cell-related "selfrenewal" signature and high epidermal growth factor receptor expression associated with resistance to concomitant chemoradiotherapy in glioblastoma. J Clin Oncol 2008;26:3015-3024.

75. Rand K, Qu W, Ho T, Clark SJ, Molloy P. Conversion-specific detection of DNA methylation using real-time polymerase chain reaction (ConLight-MSP) to avoid false positives. Methods 2002; 27:114-120.

76. Uhlmann K, Rohde K, Zeller C, et al. Distinct methylation profiles of glioma subtypes. Int J Cancer 2003;106:52-59.

77. Ohgaki H, Kleihues P. Genetic pathways to primary and secondary glioblastoma. Am J Pathol 2007;170:1445-1453.

78. Wiencke JK, Zheng S, Jelluma N, et al. Methylation of the PTEN promoter defines low-grade gliomas and secondary glioblastoma. Neuro Oncol 2007;9:271-279.

79. Hong C, Maunakea A, Jun P, et al. Shared epigenetic mechanisms in human and mouse gliomas inactivate expression of the growth suppressor SLC5A8. Cancer Res 2005;65:3617-3623.

80. Bello MJ, Martinez-Glez V, Franco-Hernandez C, et al. DNA methylation pattern in 16 tumor-related genes in schwannomas. Cancer Genet Cytogenet 2007;172:84-86.

81. Lomas J, Bello MJ, Arjona D, et al. Genetic and epigenetic alteration of the NF2 gene in sporadic meningiomas. Genes Chromosomes Cancer 2005;42:314-319.

82. Watanabe T, Katayama Y, Yoshino A, et al. Aberrant hypermethylation of p14ARF and O6-methylguanine-DNA methyltransferase genes in astrocytoma progression. Brain Pathol 2007;17: $5-10$.

83. Martinez R, Setien F, Voelter C, et al. CpG island promoter hypermethylation of the pro-apoptotic gene caspase- 8 is a common hallmark of relapsed glioblastoma multiforme. Carcinogenesis 2007;28:1264-1268.
84. Tews B, Roerig P, Hartmann C, et al. Hypermethylation and transcriptional downregulation of the CITED4 gene at $1 \mathrm{p} 34.2$ in oligodendroglial tumours with allelic losses on 1p and 19q. Oncogene 2007;26:5010-5016.

85. Reilly KM, Tuskan RG, Christy E, et al. Susceptibility to astrocytoma in mice mutant for Nf1 and Trp53 is linked to chromosome 11 and subject to epigenetic effects. Proc Natl Acad Sci U S A 2004;101:13008-13013.

86. Lee J, Son MJ, Woolard K, et al. Epigenetic-mediated dysfunction of the bone morphogenetic protein pathway inhibits differentiation of glioblastoma-initiating cells. Cancer Cell 2008;13: $69-80$.

87. Yi JM, Tsai HC, Glockner SC, et al. Abnormal DNA methylation of CD133 in colorectal and glioblastoma tumors. Cancer Res 2008;68:8094-8103.

88. Tabu K, Sasai K, Kimura T, et al. Promoter hypomethylation regulates CD133 expression in human gliomas. Cell Res 2008; 18:1037-1046.

89. Costello JF, Fruhwald MC, Smiraglia DJ, et al. Aberrant CpGisland methylation has non-random and tumour-type-specific patterns. Nat Genet 2000;24:132-138.

90. Nakamura M, Konishi N, Inui $\mathrm{T}$, et al. Genetic variations in recurrent astrocytic tumors detected by restriction landmark genomic scanning. Brain Tumor Pathol 1998;15:1-6.

91. Nakamura M, Konishi N, Tsunoda S, et al. Genomic alterations of human gliomas detected by restriction landmark genomic scanning. Brain Tumor Pathol 1997;14:13-17.

92. Nakamura M, Konishi N, Tsunoda S, et al. Analyses of human gliomas by restriction landmark genomic scanning. J Neurooncol 1997;35:113-120.

93. Nakamura M, Konishi N, Tsunoda S, et al. Genomic alterations in human glioma cell lines detected by restriction landmark genomic scanning. J Neurooncol 1997;34:203-209.

94. Costello JF, Plass C, Cavenee WK. Aberrant methylation of genes in low-grade astrocytomas. Brain Tumor Pathol 2000;17: $49-56$.

95. Meissner A, Gnirke A, Bell GW, et al. Reduced representation bisulfite sequencing for comparative high-resolution DNA methylation analysis. Nucleic Acids Res 2005;33:5868-5877.

96. Weber M, Davies JJ, Wittig D, et al. Chromosome-wide and promoter-specific analyses identify sites of differential DNA methylation in normal and transformed human cells. Nat Genet 2005;37:853-862.

97. Down TA, Rakyan VK, Turner DJ, et al. A Bayesian deconvolution strategy for immunoprecipitation-based DNA methylome analysis. Nat Biotechnol 2008;26:779-785.

98. Karpf AR, Peterson PW, Rawlins JT, et al. Inhibition of DNA methyltransferase stimulates the expression of signal transducer and activator of transcription 1,2, and 3 genes in colon tumor cells. Proc Natl Acad Sci U S A 1999;96:14007-14012.

99. Foltz G, Ryu GY, Yoon JG, et al. Genome-wide analysis of epigenetic silencing identifies BEX1 and BEX2 as candidate tumor suppressor genes in malignant glioma. Cancer Res 2006;66: 6665-6674.

100. Kongkham PN, Northcott PA, Ra YS, et al. An epigenetic genome-wide screen identifies SPINT2 as a novel tumor suppressor gene in pediatric medulloblastoma. Cancer Res 2008;68:99459953.

101. Kim TY, Zhong S, Fields CR, Kim JH, Robertson KD. Epigenomic profiling reveals novel and frequent targets of aberrant DNA methylation-mediated silencing in malignant glioma. Cancer Res 2006;66:7490-7501.

102. Mueller W, Nutt CL, Ehrich M, et al. Downregulation of RUNX3 and TES by hypermethylation in glioblastoma. Oncogene 2007; 26:583-593.

103. Bernstein BE, Mikkelsen TS, Xie X, et al. A bivalent chromatin structure marks key developmental genes in embryonic stem cells. Cell 2006;125:315-326.

104. Ohm JE, McGarvey KM, Yu X, et al. A stem cell-like chromatin pattern may predispose tumor suppressor genes to DNA hypermethylation and heritable silencing. Nat Genet 2007;39:237-242. 
105. Kanai Y, Ushijima S, Nakanishi Y, Sakamoto M, Hirohashi S. Mutation of the DNA methyltransferase (DNMT) 1 gene in human colorectal cancers. Cancer Lett 2003;192:75-82.

106. Miremadi A, Oestergaard MZ, Pharoah PD, Caldas C. Cancer genetics of epigenetic genes. Hum Mol Genet 2007;16 Spec No 1:R28-49.

107. Di Croce L. Chromatin modifying activity of leukemia associated fusion proteins. Hum Mol Genet 2005;14 Spec No 1:R77-84.

108. Bracken AP, Pasini D, Capra M, et al. EZH2 is downstream of the pRB-E2F pathway, essential for proliferation and amplified in cancer. EMBO J 2003;22:5323-5335.

109. Hayry V, Tanner M, Blom T, et al. Copy number alterations of the polycomb gene BMI1 in gliomas. Acta Neuropathol 2008; 116:97-102.

110. Tirabosco R, De Maglio G, Skrap M, Falconieri G, Pizzolitto S. Expression of the Polycomb-Group protein BMI1 and correlation with p16 in astrocytomas an immunohistochemical study on 80 cases. Pathol Res Pract 2008;204:625-631.

111. Bruggeman SW, Valk-Lingbeek ME, van der Stoop PP, et al. Ink4a and Arf differentially affect cell proliferation and neural stem cell self-renewal in Bmi1-deficient mice. Genes Dev 2005; 19:1438-1443.

112. Godlewski J, Nowicki MO, Bronisz A, et al. Targeting of the Bmi-1 oncogene/stem cell renewal factor by microRNA-128 inhibits glioma proliferation and self-renewal. Cancer Res 2008;68: 9125-9130.

113. Bruggeman SW, Hulsman D, Tanger E, et al. Bmil controls tumor development in an Ink4a/Arf-independent manner in a mouse model for glioma. Cancer Cell 2007;12:328-341.

114. Dirks P. Bmil and cell of origin determinants of brain tumor phenotype. Cancer Cell 2007;12:295-297.

115. Petrij F, Giles RH, Dauwerse HG, et al. Rubinstein-Taybi syndrome caused by mutations in the transcriptional co-activator CBP. Nature 1995;376:348-351.

116. Lucio-Eterovic AK, Cortez MA, Valera ET, et al. Differential expression of 12 histone deacetylase (HDAC) genes in astrocytomas and normal brain tissue: class II and IV are hypoexpressed in glioblastomas. BMC Cancer 2008;8:243.

117. Parsons DW, Jones S, Zhang X, et al. An integrated genomic analysis of human glioblastoma multiforme. Science 2008.

118. Saito Y, Kanai Y, Sakamoto M, et al. Overexpression of a splice variant of DNA methyltransferase 3b, DNMT3b4, associated with DNA hypomethylation on pericentromeric satellite regions during human hepatocarcinogenesis. Proc Natl Acad Sci U S A 2002;99:10060-10065.

119. Ostler KR, Davis EM, Payne SL, et al. Cancer cells express aberrant DNMT3B transcripts encoding truncated proteins. Oncogene 2007;26:5553-5563.

120. Hong C, Moorefield KS, Jun P, et al. Epigenome scans and cancer genome sequencing converge on WNK2, a kinase-independent suppressor of cell growth. Proc Natl Acad Sci U S A 2007;104: 10974-10979.

121. Moniz S, Verissimo F, Matos P, et al. Protein kinase WNK2 inhibits cell proliferation by negatively modulating the activation of MEK1/ERK1/2. Oncogene 2007;26:6071-6081.

122. Moniz S, Matos P, Jordan P. WNK2 modulates MEK1 activity through the Rho GTPase pathway. Cell Signal 2008;20:1762-1768.

123. Jun P, Hong C, Lal A, et al. Epigenetic silencing of the kinase tumor suppressor WNK2 is tumor-type and tumor-grade specific. Neuro Oncol 2008.

124. Dokmanovic M, Clarke C, Marks PA. Histone deacetylase inhibitors: overview and perspectives. Mol Cancer Res 2007;5:981-989.

125. Sathornsumetee S, Reardon DA, Desjardins A, et al. Molecularly targeted therapy for malignant glioma. Cancer 2007;110:13-24.

126. Yin D, Ong JM, Hu J, et al. Suberoylanilide hydroxamic acid, a histone deacetylase inhibitor: effects on gene expression and growth of glioma cells in vitro and in vivo. Clin Cancer Res 2007; 13:1045-1052.

127. Gui CY, Ngo L, Xu WS, Richon VM, Marks PA. Histone deacetylase (HDAC) inhibitor activation of p21WAF1 involves changes in promoter-associated proteins, including HDAC1. Proc Natl Acad Sci U S A 2004;101:1241-1246.
128. Qiu L, Kelso MJ, Hansen C, et al. Anti-tumour activity in vitro and in vivo of selective differentiating agents containing hydroxamate. Br J Cancer 1999;80:1252-1258.

129. Gray SG, Qian CN, Furge K, Guo X, Teh BT. Microarray profiling of the effects of histone deacetylase inhibitors on gene expression in cancer cell lines. Int J Oncol 2004;24:773-795.

130. Lee JH, Park JH, Jung Y, et al. Histone deacetylase inhibitor enhances 5-fluorouracil cytotoxicity by down-regulating thymidylate synthase in human cancer cells. Mol Cancer Ther 2006;5: 3085-3095.

131. Mitsiades CS, Mitsiades NS, McMullan CJ, et al. Transcriptional signature of histone deacetylase inhibition in multiple myeloma: biological and clinical implications. Proc Natl Acad Sci U S A 2004;101:540-545.

132. Peart MJ, Smyth GK, van Laar RK, et al. Identification and functional significance of genes regulated by structurally different histone deacetylase inhibitors. Proc Natl Acad Sci U S A 2005; 102:3697-3702.

133. Eyupoglu IY, Hahnen E, Buslei R, et al. Suberoylanilide hydroxamic acid (SAHA) has potent anti-glioma properties in vitro, ex vivo and in vivo. J Neurochem 2005;93:992-999.

134. Chinnaiyan P, Vallabhaneni G, Armstrong E, Huang SM, Harari PM. Modulation of radiation response by histone deacetylase inhibition. Int J Radiat Oncol Biol Phys 2005;62:223-229.

135. Chinnaiyan P, Cerna D, Burgan WE, et al. Postradiation sensitization of the histone deacetylase inhibitor valproic acid. Clin Cancer Res 2008;14:5410-5415.

136. Kumagai T, Wakimoto N, Yin D, et al. Histone deacetylase inhibitor, suberoylanilide hydroxamic acid (Vorinostat, SAHA) profoundly inhibits the growth of human pancreatic cancer cells. Int J Cancer 2007;121:656-665.

137. Ugur HC, Ramakrishna N, Bello L, et al. Continuous intracranial administration of suberoylanilide hydroxamic acid (SAHA) inhibits tumor growth in an orthotopic glioma model. J Neurooncol 2007:83:267-275

138. Entin-Meer M, Rephaeli A, Yang X, et al. Butyric acid prodrugs are histone deacetylase inhibitors that show antineoplastic activity and radiosensitizing capacity in the treatment of malignant gliomas. Mol Cancer Ther 2005;4:1952-1961.

139. Entin-Meer M, Yang X, VandenBerg SR, et al. In vivo efficacy of a novel histone deacetylase inhibitor in combination with radiation for the treatment of gliomas. Neuro Oncol 2007;9:82-88.

140. Ellis L, Pan Y, Smyth GK, et al. Histone deacetylase inhibitor panobinostat induces clinical responses with associated alterations in gene expression profiles in cutaneous T-cell lymphoma. Clin Cancer Res 2008;14:4500-4510.

141. Gimsing P, Hansen M, Knudsen LM, et al. A phase I clinical trial of the histone deacetylase inhibitor belinostat in patients with advanced hematological neoplasia. Eur J Haematol 2008;81:170 176.

142. Esteller M, Sanchez-Cespedes M, Rosell R, et al. Detection of aberrant promoter hypermethylation of tumor suppressor genes in serum DNA from non-small cell lung cancer patients. Cancer Res 1999;59:67-70.

143. Wong IH, Lo YM, Zhang J, et al. Detection of aberrant p16 methylation in the plasma and serum of liver cancer patients. Cancer Res 1999;59:71-73.

144. Weaver KD, Grossman SA, Herman JG. Methylated tumor-specific DNA as a plasma biomarker in patients with glioma. Cancer Invest 2006;24:35-40.

145. Lee J, Kotliarova S, Kotliarov Y, et al. Tumor stem cells derived from glioblastomas cultured in bFGF and EGF more closely mirror the phenotype and genotype of primary tumors than do serum-cultured cell lines. Cancer Cell 2006;9:391-403.

146. Li A, Walling J, Kotliarov Y, et al. Genomic changes and gene expression profiles reveal that established glioma cell lines are poorly representative of primary human gliomas. Mol Cancer Res 2008;6:21-30.

147. Tan J, Yang X, Zhuang L, et al. Pharmacologic disruption of Polycomb-repressive complex 2-mediated gene repression selectively induces apoptosis in cancer cells. Genes Dev 2007;21: $1050-1063$. 